\title{
EFEITO DA PODA DA ÚLTIMA PENCA DO CACHO DA BANANEIRA PRATA ANÃ (AAB) IRRIGADA NA PRODUÇÃO DE FRUTOS NO NORTE DE MINAS GERAIS ${ }^{1}$
}

\author{
MARIA GERALDA V. RODRIGUES ${ }^{2}$, ROSILENE FERREIRA SOUTO³, JOÃO L. P. MENEGUCCI ${ }^{4}$
}

\begin{abstract}
RESUMO - Este trabalho foi conduzido na Fazenda Experimental de Mocambinho pertencente à EPAMIG/CTNM, localizada no Perímetro Irrigado do Jaíba MG, com o objetivo de avaliar o efeito da retirada da última penca do cacho de banana 'Prata Anã', na precocidade de colheita e características dos frutos. O delineamento experimental utilizado foi inteiramente casualizado, com 2 tratamentos, 23 repetições e 3 plantas por parcela. Os tratamentos foram representados por retirada e manutenção da última penca. Não houve efeito dos tratamentos para precocidade, nos dois primeiros ciclos de produção. Para os parâmetros de produção, não houve diferença estatística nos dois primeiros ciclos para massa do cacho; massa média de frutos; massa média de pencas; comprimento, diâmetro, massa e espessura de casca do fruto central da segunda penca; massa da primeira penca; massa da segunda penca; massa da penúltima penca e massa do engaço. No segundo ciclo, houve efeito negativo da retirada da última penca para massa total de frutos, e no primeiro ciclo, apesar de não ter sido significativo, observou-se a mesma tendência. No terceiro ciclo a retirada da última penca promoveu aumento de massa média de frutos, massa média de pencas e diâmetro do fruto central da segunda penca, não alterando o massa total de frutos.
\end{abstract}

Temos para indexação: Musa sp., poda de penca, Prata Anã, qualidade de frutos.

\section{INFLUENCE OF LAST HAND PRUNE ON THE IRRIGATED BANANA BUNCH CROP IN THE NORTH OF MINAS GERAIS STATE}

\begin{abstract}
This work was carried out in the EPAMIG/CTNM's Experimental Farm, wich is located in the irrigated area of Jaiba county in the north of Minas Gerais state. The aim of this work was to evaluate the effect of hand prune on the harvest time and on the crop characteristics. A completely randomized design was used with 23 replications and three plants per plot. The treatments were pruning and nonpruning of last hand of each bunch. There was no effect of treatments on the harvest time for two first crops, and on fruits characteristics. In the second crop cycle, total fruit weight decreased with last hand prunning. In the third crop the last hand prune treatment increased the mean weight of fuits and of the hands, and the diameter of the central finger of the second hand, but there was no effect in the total fruit weight
\end{abstract}

Index terms: Musa sp., hand prune, Prata Anã, fruit quality.

\section{INTRODUÇÃO}

A bananicultura do Norte de Minas necessita de melhorias, principalmente no que diz respeito a qualidade dos frutos, uma vez que a remuneração dos frutos classificados como de segunda é hoje $50 \%$ da remuneração referente aos frutos de primeira. Esta diferença de preço justifica um investimento em pesquisas de técnicas que possam garantir o retorno dos grandes investimentos feitos na região.

Segundo Soto Ballestero (1992), a poda de mãos foi idealizada pelos técnicos e produtores de banana, para eliminar as mãos inferiores cujos dedos não atingem as dimensões exigidas pelo mercado internacional. Para tanto se fixou como norma, a retirada de uma ou duas pencas, para cachos com nove ou mais pencas respectivamente, para variedades do subgrupo Cavendish. Em clones de dedos curtos como 'Gran Enano' e 'Dwarf Cavendish', a poda pode ser ainda maior.

A última penca do cacho é, em geral, defeituosa, com bananas muito curtas e por isso tais pencas são descartadas durante a embalagem em caixas (Moreira, 1987 e Souto et al 1997).

O despencamento consiste em eliminar esta última penca, deixando-se apenas um fruto, que permitirá a circulação de seiva, evitando o ataque de doenças (Moreira, 1987; Borges et al, 1997 e Souto et al 1997). Esta operação faz com que todas as bananas das demais pencas tenham aumento de tamanho e diâmetro (Moreira, 1987; Soto Ballestero 1992 e Souto et al 1997), além de melhoria de qualidade dos frutos (Lichtemberg et al, 1991 e Soto, 1992).

Boncato (1977), trabalhando com a 'Lacatan', comparou os tratamentos em que reduziu o número de pencas por cacho para $4,5,6$ ou 7 , com o tratamento em que deixou todas se desenvolverem. $\mathrm{O}$ tratamento com menor número de pencas por cacho resultou num aumento do massa média das pencas e dos frutos, bem como no aumento do tamanho e da circunferência dos frutos.

Este trabalho foi conduzido com o objetivo de avaliar o

1 (Trabalho 171/2000). Recebido: 03/08/2000. Aceito para publicação: 08/02/2002.

2 Eng. Agro, MS., Pesquisadora EPAMIG/CTNM Janaúba-MG, magevr@hotmail.com

3 Eng. Agro, MS., Pesquisadora EPAMIG/CTTP Uberaba-MG, rfsouto@hotmail.com

4 Eng. Agro Dr., Pesquisador EMBRAPA/Mandioca e Fruticultura/ SNT Uberlândia-MG, meneguci@cnpmf.embrapa.br 
efeito da retirada da última penca de frutos do cacho de banana Prata Anã, na precocidade de colheita e qualidade dos frutos.

\section{MATERIAL E MÉTODOS}

O trabalho foi conduzido na Fazenda Experimental de Mocambinho (FEMO) pertencente à EPAMIG (CTNM), no Projeto Jaíba . O Projeto Jaíba está localizado no extremo norte do Estado de Minas Gerais entre 43 $29^{\prime}$ e $44^{\circ} 6^{\prime}$ de longitude Oeste, $14^{\circ} 33^{\prime}$ e $15^{\circ} 28^{\prime}$ de latitude sul com altitude de aproximadamente $500 \mathrm{~m}$. Possui pluviosidade média anual de $871 \mathrm{~mm}$, concentrados de novembro a março. A temperatura média anual é de $24{ }^{\circ} \mathrm{C}$ e as médias de verão e inverno são 32 e $19,5^{\circ} \mathrm{C}$ respectivamente. A insolação é de 2763 horas anuais e umidade relativa média de $70,6 \%$, sendo que no período seco a UR pode chegar a extremos de $20 \%$.

O solo da unidade foi classificado como Latossololo Vermelho Amarelo Distrófico, apresentando na camada de 0 a $20 \mathrm{~cm}$, classe textural franco argilo arenoso, com $22 \%$ de argila, $68 \%$ de areia e $10 \%$ de silte, cultivado pela primeira vez. Foi feita análise de amostra do solo coletada antes do preparo da área, que apresentou $\mathrm{pH}$ em água de $4,0 / \mathrm{dm}^{3}$, baixos teores de fósforo $(1,8 \mathrm{ppm})$, potássio $(57 \mathrm{ppm})$, cálcio $\left(1,1 \mathrm{meg} / 100 \mathrm{~cm}^{3}\right)$, magnésio $\left(0,4 \mathrm{meg} / 100 \mathrm{~cm}^{3}\right)$, alumínio $\left(0,5 \mathrm{meg} / 100 \mathrm{~cm}^{3}\right)$ e $1,4 \%$ de matéria orgânica.

A área foi preparada com aração seguida das devidas correções, com calagem, gradagem e preparo dos sulcos com 30 $\mathrm{cm}$ de profundidade. Adicionou-se 3,2 litros de esterco de galinha por metro linear de sulco. As covas de 40x40x40cm foram abertas sobre os sulcos, com adição de fosfato e micronutrientes.

As mudas obtidas por cultura de tecidos, foram repicadas para sacos de polietileno onde permaneceram por 30 dias e então levadas a campo e plantadas no espaçamento $3.0 \mathrm{x}$ $2.7 \mathrm{~m}$. As adubações de cobertura foram realizadas mensalmente de acordo com a necessidade da cultura, e as análises de solo e folhas realizadas a cada 4 meses.
A irrigação foi realizada com aspersão convencional nos primeiros 45 dias, sendo então substituída por microaspersão. Como o solo possui baixa capacidade de retenção de água, a irrigação foi feita diariamente, calculando-se a lâmina de água com base na evaporação do Tanque Classe A e Kc da cultura. Utilizou-se Kc recomendado por Doorembos e Kassam (1994), porém mantendo o valor 1 , à partir do oitavo mês. Todos os dados climáticos necessários ao cálculo da irrigação, foram obtidos na estação meteorológica do INMET localizada na FEMO.

$\mathrm{O}$ delineamento experimental utilizado foi inteiramente casualizado, com 2 tratamentos, 23 repetições e 3 plantas por parcela. Os tratamentos foram representados por poda da última penca e sem poda da última penca. Nas plantas avaliadas a retirada da última penca foi feita quando esta se voltava para cima, sendo que uma das duas fileiras de frutos se encontrava na horizontal. As mesmas parcelas foram avaliadas durante os três ciclos de produção.

A colheita foi feita segundo a metodologia tradicionalmente utilizada na região, quando os frutos apresentavam mudanças da tonalidade verde escuro para verde claro e redução das quinas.

Foi feito controle de broca-do-rizoma utilizando-se iscas do tipo queijo, para monitoramento da incidência e controle químico quando necessário. A sigatoka amarela foi monitorada através da incidência de manchas nas folhas, para definição da época e da utilização do controle químico .

Foram avaliadas as variáveis relacionadas na Tabela 1. Foi feita análise estatística dos dados e análise das médias pelo teste de Tukey.

\section{RESULTADOS E DISCUSSÃO}

Após fixadas as parcelas as plantas foram acompanhadas individualmente, sendo tomadas as datas de floração. As plantas do primeiro ciclo floresceram de 20/01 a 13/ 02/1997. No segundo ciclo o florescimento ocorreu de 25/03 a 14/

TABELA 1 - Efeito da poda de última penca no primeiro ciclo de produção da banana Prata Anã, cultivada sob irrigação no Norte de Minas Gerais, 1996.

\begin{tabular}{|c|c|c|c|c|c|c|c|c|c|c|c|c|c|c|c|}
\hline \multirow{3}{*}{$\begin{array}{l}\text { Parâmetros avaliados } \\
\text { Ponto de retirada da última penca à colheita (dias) }\end{array}$} & \multicolumn{5}{|c|}{ Primeiro Ciclo } & \multicolumn{5}{|c|}{ Segundo Ciclo } & \multicolumn{5}{|c|}{ Terceiro Ciclo } \\
\hline & \multicolumn{2}{|c|}{ Compenca } & \multicolumn{2}{|c|}{ Sempenca } & \multirow{2}{*}{$\begin{array}{l}\mathrm{CV} \\
6,99\end{array}$} & \multicolumn{2}{|c|}{ Compenca } & \multicolumn{2}{|c|}{ Sempenca } & \multirow{2}{*}{\begin{tabular}{l|}
$\mathrm{CV}$ \\
7,90
\end{tabular}} & \multicolumn{2}{|c|}{ Compenca } & \multicolumn{2}{|c|}{ Sempenca } & \multirow{2}{*}{$\begin{array}{l}\text { CV } \\
10,11\end{array}$} \\
\hline & 126,77 & $\mathrm{a}$ & 125,83 & $\mathrm{a}$ & & 138,00 & $\mathrm{a}$ & 135,00 & a & & 123,00 & $\mathrm{a}$ & 127,00 & $\mathrm{a}$ & \\
\hline Peso do cacho $(\mathrm{Kg})$ & 13,51 & a & 12,77 & $\mathrm{a}$ & 15,63 & 15,04 & $\mathrm{a}$ & 14,51 & b & 10,82 & 20,82 & $\mathrm{a}$ & 21,46 & $\mathrm{a}$ & 16,29 \\
\hline Número de pencas & 8,58 & a & 8,03 & b & 5,04 & 8,54 & $\mathrm{a}$ & 7,85 & $\mathrm{a}$ & 6,68 & 10,49 & $\mathrm{a}$ & 9,87 & b & 6,11 \\
\hline Peso total de frutos $(\mathrm{Kg})$ & 11,28 & a & 10,42 & $\mathrm{a}$ & 10,56 & 13,25 & $\mathrm{a}$ & 12,37 & $\mathrm{a}$ & 11,24 & 18,49 & $\mathrm{a}$ & 19,08 & $\mathrm{a}$ & 16,57 \\
\hline Peso médio de frutos (g) & 91,05 & $\mathrm{a}$ & 89,36 & a & 9,98 & 109,66 & $\mathrm{a}$ & 111,78 & $\mathrm{a}$ & 9,67 & 108,60 & $\mathrm{~b}$ & 118,70 & $\mathrm{a}$ & 13,98 \\
\hline Peso médio de pencas $(\mathrm{Kg})$ & 1,31 & a & 1,30 & $\mathrm{a}$ & 9,35 & 1,55 & a & 1,53 & $\mathrm{a}$ & 15,97 & 1,75 & b & 1,92 & $\mathrm{a}$ & 16,20 \\
\hline Comprimento do fruto central da $2^{\mathrm{a}}-$ penca $(\mathrm{cm})$ & 13,15 & a & 13,12 & $\mathrm{a}$ & 4,78 & 14,90 & $\mathrm{a}$ & 14,95 & $\mathrm{a}$ & 5,20 & 13,52 & $\mathrm{a}$ & 13,82 & $\mathrm{a}$ & 6,74 \\
\hline Diâmetro do fruto central da 2- penca $(\mathrm{cm})$ & 3,27 & a & 3,24 & $\mathrm{a}$ & 2,78 & 3,40 & a & 3,41 & $\mathrm{a}$ & 3,50 & 3,24 & $\mathrm{~b}$ & 3,34 & $\mathrm{a}$ & 4,66 \\
\hline Peso do fruto central da $2^{-}-$penca $(\mathrm{g})$ & 107,86 & $\mathrm{a}$ & 103,88 & $\mathrm{a}$ & 11,95 & 121,96 & a & 122,82 & $\mathrm{a}$ & 9,11 & 122,10 & $\mathrm{a}$ & 128,42 & $\mathrm{a}$ & 14,05 \\
\hline Espessura casca fruto central da $2^{\mathrm{a}-}$ penca $(\mathrm{cm})$ & 0,31 & $\mathrm{a}$ & 0,30 & $\mathrm{a}$ & 8,37 & 0,34 & $\mathrm{a}$ & 0,30 & $\mathrm{a}$ & 6,22 & 0,30 & $\mathrm{a}$ & 0,29 & $\mathrm{a}$ & 4,76 \\
\hline Peso da penca $1(\mathrm{~kg})$ & 1,21 & a & 1,18 & $\mathrm{a}$ & 8,14 & 1,89 & $\mathrm{a}$ & 1,92 & $\mathrm{a}$ & 19,96 & 2,49 & $\mathrm{a}$ & 2,47 & $\mathrm{a}$ & 23,66 \\
\hline Peso da penca $2(\mathrm{Kg})$ & 1,68 & $\mathrm{a}$ & 1,63 & $\mathrm{a}$ & 10,78 & 1,73 & $\mathrm{a}$ & 1,80 & $\mathrm{a}$ & 11,51 & 2,07 & $\mathrm{a}$ & 2,24 & $\mathrm{a}$ & 19,48 \\
\hline Número de frutos da penúltima penca & 13,86 & a & 13,74 & $\mathrm{a}$ & 3,05 & 13,38 & $\mathrm{a}$ & 13,24 & $\mathrm{a}$ & 4,67 & 15,44 & $\mathrm{a}$ & 15,56 & $\mathrm{a}$ & 3,09 \\
\hline Peso da penúltima penca $(\mathrm{Kg})$ & 1,19 & a & 1,20 & a & 8,14 & 1,28 & $\mathrm{a}$ & 1,36 & $\mathrm{a}$ & 8,01 & 1,42 & $\mathrm{a}$ & 1,56 & $\mathrm{a}$ & 15,68 \\
\hline Número de frutos da última penca & 11,67 & b & 13,68 & $\mathrm{a}$ & 9,91 & 11,55 & b & 13,07 & $\mathrm{a}$ & 13,02 & 12,25 & b & 15,62 & $\mathrm{a}$ & 13,94 \\
\hline Peso da última penca $(\mathrm{Kg})$ & 0,89 & $\mathrm{~b}$ & 1,12 & $\mathrm{a}$ & 16,38 & 1,05 & $\mathrm{~b}$ & 1,29 & $\mathrm{a}$ & 18,00 & 0,99 & $\mathrm{~b}$ & 1,49 & $\mathrm{a}$ & 19,90 \\
\hline Peso do engaço $(\mathrm{Kg})$ & 1,69 & $\mathrm{a}$ & 1,54 & $\mathrm{a}$ & 13,92 & 1,45 & $\mathrm{a}$ & 1,45 & $\mathrm{a}$ & 17,77 & 2,33 & $\mathrm{a}$ & 2,38 & $\mathrm{a}$ & 17,28 \\
\hline
\end{tabular}

As médias seguidas pela mesma letra na mesma linha, não diferem ente si pelo teste de Tukey ao nível de 5\%. 
10/1997. Já no terceiro ciclo, o período de floração foi compreendido entre 21/11/1997 a 23/01/1998.

A colheita feita segundo a metodologia descrita, foi realizada no período de $26 / 05$ a 26/06/1997 para o primeiro ciclo, de 16/09/1997 a 09/02/1998 para o segundo e de 08/04 a 28/10/ 1998 para o terceiro.

Avaliados os três primeiros ciclos de produção, foram observados os resultados encontrados na tabela 1 .

Não houve efeito de tratamento para precocidade, representada pelo período entre o momento de retirada da última penca e o ponto de colheita, nos três primeiros ciclos de produção, discordando do observado por Lichtemberg et al (1991), em trabalho feito com a 'Nanicão'. Esta diferença de resultados provavelmente ocorreu em função dos trabalhos terem sido conduzidos com diferentes variedades. Outra diferença foi na metodologia utilizada, enquanto Lichetemberg et al (1991) utilizaram cachos lançados numa só época, neste trabalho as parcelas foram fixadas aleatoriamente, por ocasião do plantio, e portanto a floração ocorreu em datas diferentes.

Para as variáveis de produção, não houve diferença estatística nos dois primeiros ciclos para massa do cacho; massa média de frutos; massa média de pencas; comprimento, diâmetro, massa e espessura de casca do fruto central da segunda penca; massa penca 1; massa penca 2; massa da penúltima penca e massa do engaço. Estes resultados contrariam as descrições feitas por Soto (1992); Moreira (1987) e Boncato (1970). No segundo ciclo de colheita houve efeito negativo da retirada de penca para massa total de frutos.

No terceiro ciclo a retirada da última penca promoveu aumento de massa média de frutos, massa média de pencas e diâmetro do fruto central da segunda penca, não alterando o massa total de frutos.

Conforme esperado, a retirada da última penca reduziu o número de pencas do cacho. Da mesma forma, aumentou o número de frutos e massa da última penca, conforme observado por Lichtemberg (1991), que diz que a última penca remanescente apresentou frutos maiores no tratamento com poda, cuja penca avaliada era a penúltima, enquanto que no tratamento sem poda, a penca avaliada foi a última.

\section{CONCLUSÕES}

A poda da última penca não teve efeito sobre a produção de frutos nos dois primeiros ciclos.

Para as variáveis de produção, não houve diferença estatística nos dois primeiros ciclos, variando apenas o número de pencas e de frutos da última penca, e a massa da última penca. No segundo ciclo, houve efeito negativo da retirada de penca para massa total de frutos.

No terceiro ciclo a retirada da última penca promoveu aumento de massa média de frutos, massa média de pencas e diâmetro do fruto central da segunda penca, não alterando o massa total de frutos.

Conforme esperado, a retirada da última penca reduziu o número de pencas do cacho. Da mesma forma, aumentou o número de frutos e massa da última penca.

\section{REFERÊNCIAS BIBLIOGRÁFICAS}

BONCATO, A. A.; Effects of reducing the number of hands in a bunch of Lacatan banana. Phillip. J. Plant Ind., Phillippine, 32:24351. 1967. 1:Horticultural Abstracts, England 40 (2): 580, Abstr. 4844. 1970.

BORGES, A L.; ALVES, E. J.; SILVA, S . de O; SOUZA, L. da S.; MATOS, A P. de; FANCELLI, M.; OLIVEIRA, A M. G.; CORDEIRO, Z. J. M.; SILEIRA, J. R. S.; COSTA, D. da C.; MEDINA, V. M.; OLIVEIRA, S. L. de; SOUZA, J. das S. OLIVEIRA, R. P. de; CARDOSO, C. E. L.; MATSUURA, F.C. A U.; ALMEIDA, C. O de. O Cultivo da Banana. Cruz das Almas: EMBRAPACNPMF, 1997. 109 P. (EMBRAPA-CNPMF. Circular Técnica, 27).

DOORENBOS,J.; KASSAM,A.H. Efeito da água no rendimento das culturas. Campina Grande: UFPB, 1994. 306p. (FAO. Irrigação e Drenagem, 33).

LICHTEMBERG, L. A.; ZAFFARI, G. R. e HINZ, R. H. Experimentos preliminares sobre poda da inflorescência masculina e poda de pencas em banana 'Nanicão'. Rev. Bras. Frutic. Cruz das Almas, v.13, n.2, out. 1991. p.9-18.

MOREIRA, R. S. Banana: Teoria e Prática de Cultivo. Campinas, Fundação Cargill, 1987.335 p.

SOTO BALLESTERO, M. Bananos: cultivo y comercialización. 2.ed. San José, Costa Rica: Litografia e Imprenta Lil, 1992. 674 p.

SOUTO, R. F; RODRIGUES, M. G. V.; ALVARENGA, C.D.; SILVA, J. T. A.da; MAENO, P.; GONZAGA, V. Sistema de Produção para a Cultura da Banana Prata- Anã, no Norte de Minas. Belo Horizonte: EPAMIG, 1997. 32 p. (EPAMIG. Boletim Técnico, 48). 